\begin{tabular}{|c|l|}
\hline Title & A nalyses of trajectory on-the fly based on the global reaction route map \\
\hline Author(s) & Tsutsumi, Takuro; Harabuchi, Yu; Ono, Y uriko; Maeda, Satoshi; T aketsugu, Tetsuya \\
\hline Citation & $\begin{array}{l}\text { Physical chemistry chemical physics, 20(3), 1364-1372 } \\
\text { https://doi.org/40.1039/c7cp06528k }\end{array}$ \\
\hline Issue Date & 2018 01-21 \\
\hline Doc URL & http://hdl.handle.net/2115/72346 \\
\hline Type & article(author version) \\
\hline File Information & PCCP20-31364-1372.pdf \\
\hline
\end{tabular}

Instructions for use 


\title{
Analyses of trajectory on-the-fly based on the global reaction route map
}

\author{
Takuro Tsutsumi, ${ }^{a}$ Yu Harabuchi,,${ }^{a, b}$ Yuriko Ono, ${ }^{a}$ Satoshi Maeda, ${ }^{a}$ \\ and Tetsuya Taketsugu ${ }^{\mathrm{a}, *}$ \\ ${ }^{a}$ Department of Chemistry, Faculty of Science, Hokkaido University, Sapporo 060-0810, Japan \\ ${ }^{b}$ Precursory Research for Embryonic Science and Technology (PRESTO), Japan Science and \\ Technology Agency (JST), Saitama 332-0012, Japan.
}

\begin{abstract}
A methodology to analyze a trajectory on-the-fly (TOF) based on a global reaction route map consisting of intrinsic reaction coordinate (IRC) pathways is proposed. By using the distance functions in the configurational space, the location of each point on trajectories is detected, providing a dynamical picture that the molecular system goes over several minima and transition states in the reaction path network. In the application to structural transformations of $\mathrm{Au}_{5}$ cluster, a variety of reaction routes are obtained, and the hopping from one IRC to the other IRC (IRC-jump) is analyzed. The branching of trajectories over many minima on the potential energy surface via valley-ridge transition points is also discussed.
\end{abstract}

\section{KEYWORDS}

Trajectory on-the-fly, bifurcation, reaction pathways, gold cluster, nuclear permutation-inversion isomer

\section{AUTHOR INFORMATION}

Corresponding Author: E-mail: take@sci.hokudai.ac.jp (T. Taketsugu) 


\section{INTRODUCTION}

In last two decades, the trajectory on-the-fly molecular dynamics (TOF-MD) such as ab initio molecular dynamics (AIMD) ${ }^{1-3}$ and Car-Parrinello Molecular dynamics ${ }^{4}$ without potential functions has become one of standard quantum chemical approaches to examine reaction mechanisms and dynamics of various types of chemical reactions. TOF-MD is a full-dimensional classical trajectory method which uses atomic forces derived from a wave function theory ${ }^{1-3,5}$ or a density functional theory (DFT) ${ }^{4}$ calculation, and thus the molecular system could reach several isomer structures or dissociated fragments within a given energy. In TOF-MD simulations, one can examine reaction dynamics of the molecules without any assumption for reaction pathways and products, which could provide knowledge about the significant internal degrees of freedom and unknown reaction pathways. The target of TOF-MD is now extended to photo-reactions such as a photoisomerization or an ultrafast relaxation process of excited-molecules. ${ }^{6-8}$

In quantum chemical study for reactions, an intrinsic reaction coordinate (IRC) ${ }^{9,10}$ is a useful concept to describe the reaction pathway for an elementary reaction. The IRC is defined as a minimum energy pathway in mass-weighted coordinates, which connects one transition state (TS) and two minima (MINs) on a potential energy surface (PES). The changes of the molecular structure and adiabatic potential energy along the IRC pathway can provide an intuitive picture of the reaction process, and also variations of the electronic wavefunction along the pathway provides insights to the reaction mechanism. In the actual reactions, however, the molecule with a kinetic energy can deviate from the IRC pathway. ${ }^{3,11}$ In the TOF-MD simulations, one can examine dynamical effects such as a centrifugal force due to a path curvature and a branching of the products. In a previous study, one of the authors proposed a method to analyze a trace of the AIMD trajectory based on a single IRC pathway where the trajectory is represented by a reaction coordinate, a curvature coordinate, and a deviation from the two-dimensional reaction plane along the IRC pathway. ${ }^{12,13}$ 
In 2004, Ohno and Maeda proposed an automated reaction path search method, ${ }^{14}$ referred to as anharmonic downward distortion following (ADDF), ${ }^{15,16}$ which can locate transition state geometries systematically from a given minimum based on a geometrical feature of the PES. A combined use of ADDF and IRC computations lead to the concept of an automated reaction path search that can generate a global reaction route map for a given molecular system. Such an approach makes it possible to predict new reaction pathways without any assumptions on the reaction mechanism. Recently, the single component - artificial force induced reaction (SC-AFIR) method has been developed as an efficient reaction-path search method, and been used in many applications. ${ }^{16,17}$ The recent development in an automated reaction path search method is reviewed in ref 17.

In this study, we propose a novel methodology to analyze TOF based on a global reaction route map generated by ADDF and IRC computations. The global reaction route map provides a static information of the reaction pathways on the PES, while TOF can go around over the global reaction route map dynamically. Through the present analyses, one can discuss dynamical aspect of the reaction routes based on the static reaction-path network. The analyses were applied to structural transformations of a small gold cluster, $\mathrm{Au}_{5}$, for which we reported detailed analyses of a global reaction route map, ${ }^{18}$ including branching pathways caused by a valley-ridge transition (VRT) points on the IRC. ${ }^{19,20}$ 


\section{METHODOLOGY}

First, a global reaction route map is generated by ADDF and IRC computations for a given molecular system. Second, TOF-MD simulations are executed at the same computational level, which provide a bunch of trajectories on the full-dimensional PES. In TOF-MD simulations, a specific reaction is not assumed and all degrees of freedom are taken into account automatically, and thus various reactions can occur within a given energy. Third, the TOF is analyzed based on the global reaction route map. To map the TOF into the IRC pathways, we introduce a distance function, $d_{i}(t)$, defined as

$$
d_{i}(t)=\left|\mathbf{x}^{\mathrm{TOF}}(t)-\mathbf{x}_{i}^{\mathrm{IRC}}\right|
$$

where $\mathbf{x}^{\mathrm{TOF}}(t)$ and $\mathbf{x}_{i}^{\mathrm{IRC}}$ denote $3 N$-dimensional mass-weighted Cartesian coordinates for a point on the TOF at a time $t\left(\mathbf{x}^{\mathrm{TOF}}(t)\right)$ and the $i$ th reference point on the IRC networks $\left(\mathbf{x}_{i}^{\mathrm{IRC}}\right)$, respectively, for an $N$ atomic system. When calculating $d_{i}(t)$ in Eq. (1), the origin of the coordinate system is set to the center of mass, and the $\mathrm{x}-\mathrm{y}-\mathrm{z}$ coordinate axes for $\mathbf{x}^{\mathrm{TOF}}(t)$ and $\mathbf{x}_{i}^{\mathrm{IRC}}$ are determined so as to minimize $d_{i}(t)$, using the Kabsch algorithm. ${ }^{21}$ For a given point $\mathbf{x}^{\mathrm{TOF}}(t)$, the point $\mathbf{x}_{i}^{\mathrm{IRC}}$ with the smallest $d_{i}(t)$ is regarded as the closest IRC point (referred to as $\mathbf{x}^{\mathrm{IRC}}(t)$ ), and the smallest $d_{i}(t)$ is referred to as $d(t)$ as a distance from the IRC path. According to this analysis, one can discuss the situation where the molecule jumps from one IRC to another IRC during the structural transformations. Figure 1 shows a schematic picture where a TOF runs over the reaction route map. In this case the molecule jumps from the IRC that connects TS0 and MIN0 to the other IRC that connects TS1 and MIN1. 


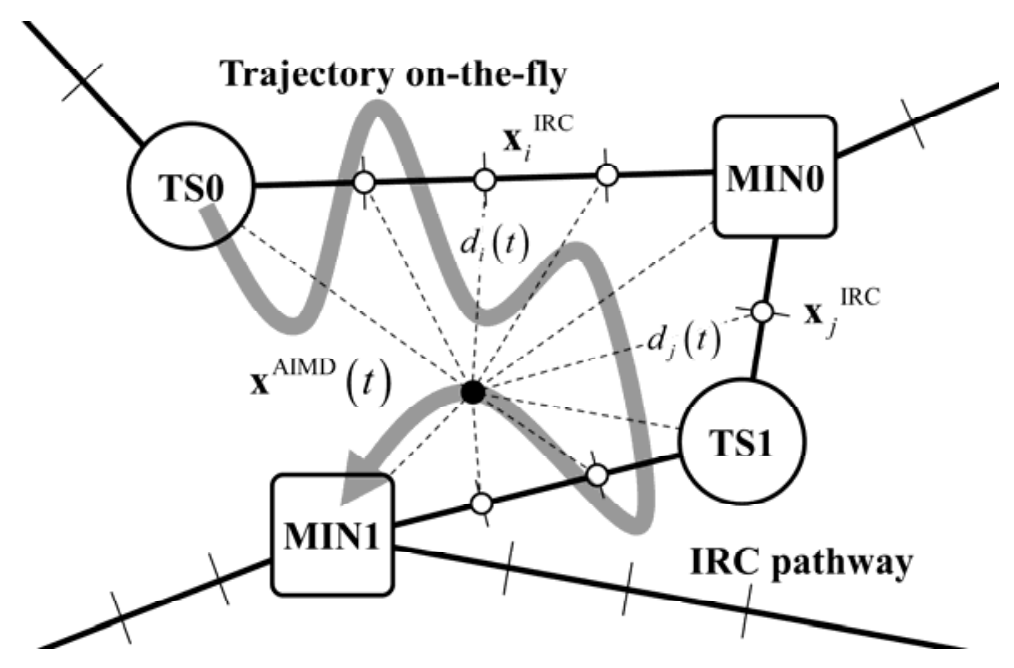

Figure 1. A schematic illustration of a TOF that jumps from one IRC to another IRC.

As the number of reference points on the IRC pathways increases, the present analyses become more informative, and simultaneously complicated. If one wants to discuss the branching ratio over a lot of product minima, it is sufficient to include only minima as reference points, and there are similar approaches in the molecular dynamics field which considers only minima. If one wants to discuss reaction dynamics relative to the IRC pathways, several points along the IRC pathways should be included as reference points. In the present application to $\mathrm{Au}_{5}$, we included not only minima but also TSs and VRT points as reference points.

In the global reaction route map determined by ADDF and IRC computations, the respective minima and TS structures are distinguished by a set of interatomic distances, and thus nuclear permutation-inversion (NPI) isomers are not distinguished each other because these isomers have the same set of interatomic distances. In TOF-MD simulations, however, these NPI isomers should appear as different structures in a configurational space. To discuss the branching process over NPI isomers, we need to distinguish the NPI-isomers. ${ }^{22,23}$ 


\section{RESULTS AND DISCUSSION}

\section{III-A. The global reaction route map for $\mathrm{Au}_{5}$ cluster}

Very recently, we reported ${ }^{18}$ a global reaction route map for $\mathrm{Au}_{5}$, generated by a combined use of ADDF and IRC computations based on DFT calculations with Perdew-Burke-Ernzerhof (PBE) functionals and LanL2DZ basis sets, using Gaussian $09^{24}$ and the developmental version of GRRM. ${ }^{25}$ Figure 2 shows the global reaction route map for $\mathrm{Au}_{5}$ where five MINs (MIN $i ; i=1 \sim 5$ ) are connected via 14 TSs (TSi-j which connects MINi and MINj). Among 14 TSs, seven TSs are categorized as TSi-i that connects two NPI isomers of MINi, indicated by a double line between MINi and TSi-i. The global minimum, MIN1, is connected with nine TSs, consisting of four TSi-i (TS1-1a, TS1-1b, TS1-1c, TS1-1d) and five TSi-j (TS1-2, TS1-3a, TS1-3b, TS1-4, TS1-5). It is noted that TS1-3a and TS1-3b are independent transition states that connect MIN1 and MIN3. As shown in Fig. 2, most MINs and TSs have a planar structure due to a relativistic effect of gold atoms, ${ }^{26}$ and only MIN4, TS1-1d, TS1-4, TS4-4, and TS5-5 have a non-planar geometry.

In Fig. 2, geometries at the valley-ridge transition (VRT) points are also shown in a black square. ${ }^{18}$ The VRT points are related to a bifurcation of the reaction pathway where the pathway of a valley nature on the PES branches into two valleys due to valley-ridge transition along the IRC pathway. ${ }^{27-34}$ Of course, the IRC pathway itself cannot bifurcate on the way by its definition as a steepest descent path, and one can recognize only an appearance of a VRT point by the vibrational analyses of the transverse normal modes along the IRC pathways. Recently, we discovered that appearance of the VRT point is a sign of the existence of the TS that connects two branching product minima; this finding was confirmed by TOF-MD simulations. ${ }^{18}$ In an $\mathrm{Au}_{5}$ case, we located five VRTs (VRTi; $i=1 \sim 5$ ) where VRT1, VRT2, VRT3, VRT4, and VRT5 correspond to TS1-1b, TS1-1c, TS5-5, TS3-3, and TS3-3, respectively. 


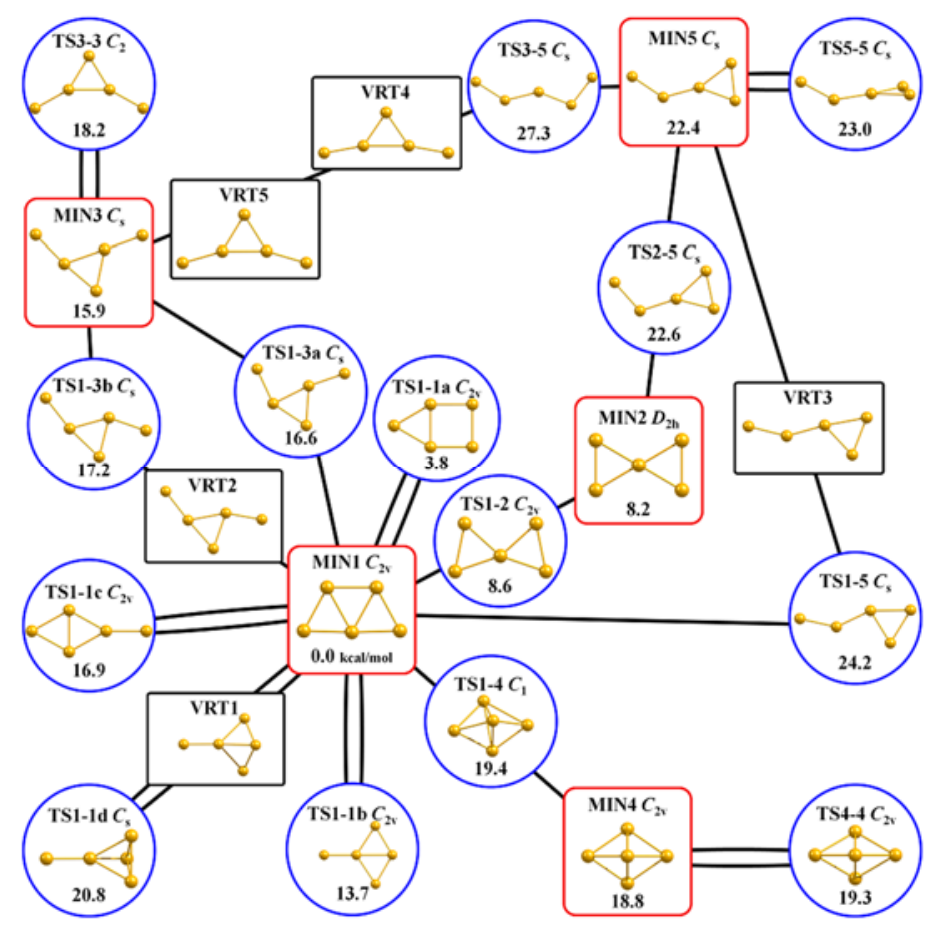

Figure 2. The global reaction route map of $\mathrm{Au}_{5}$ in Ref. 18. Five MINs (in red square) and 14 TSs (in blue circle) are connected by the IRC pathways. The point group and relative energy (in $\mathrm{kcal} / \mathrm{mol}$ ) are given for each structure. Five valley-ridge transition (VRT) points (in black square) are also shown.

As mentioned above, NPI isomers are not distinguished in the global reaction route map in Fig. 2. In the case of an $\mathrm{Au}_{5}$ cluster, the number of the NPI isomers of the $C_{1}$ structure (TS1-4) is 240 $\left(=2 \times 5\right.$ !). This number is reduced to 120 for the $C_{\mathrm{s}}$ and $C_{2}$ structures (MIN3, MIN5, TS1-1d, TS1-3a, TS1-3b, TS1-5, TS2-5, TS3-3, TS3-5, TS5-5), 60 for the $C_{2 \mathrm{v}}$ structure (MIN1, MIN4, TS1-1a, TS1-1b, TS1-1c, TS1-2, TS4-4), and 30 for the $D_{2 \mathrm{~h}}$ structure (MIN2). The respective NPI isomers of MIN1 could be transformed each other in a configurational space. As shown in Fig. 2, there are four TSs which connect NPI isomers of MIN1 each other, i.e., TS1-1a (the activation 
barrier $=3.8 \mathrm{kcal} / \mathrm{mol})$, TS1-1b $(13.7 \mathrm{kcal} / \mathrm{mol})$, TS1-1c $(16.9 \mathrm{kcal} / \mathrm{mol})$, and TS1-1d $(20.8 \mathrm{kcal} / \mathrm{mol})$.

Figure 3 shows the NPI isomers connected by the respective TSs. As shown here, five NPI isomers are connected through a structural transformation via TS1-1a, indicating that 60 NPI isomers of MIN1 are divided to 12 groups $(60 / 5=12)$. In the same way, four, six, and two NPI isomers of MIN1 are connected via TS1-b, TS1-1c and TS1-1d, respectively. 60 NPI isomers of MIN1 are completely connected by a combination of two sets of TSs as (TS1-1a and TS1-1b), (TS1-1a and TS1-1c), (TS1-1a and TS1-1d) and (TS1-1b and TS1-1c), while in the combination of TS1-1b and TS1-1d, only 12 NPI isomers are connected with each other. Two NPI isomers connected by TS1-1d are included in six NPI isomers connected by TS1-1c.

(a) The connectivity of TS1-1a

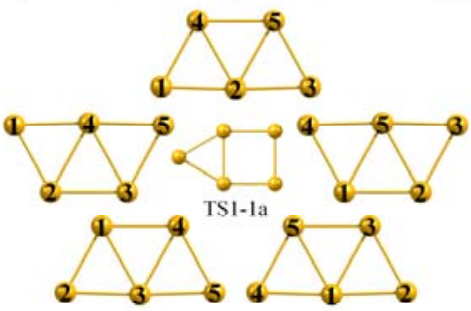

(c) The connectivity of TS1-1c

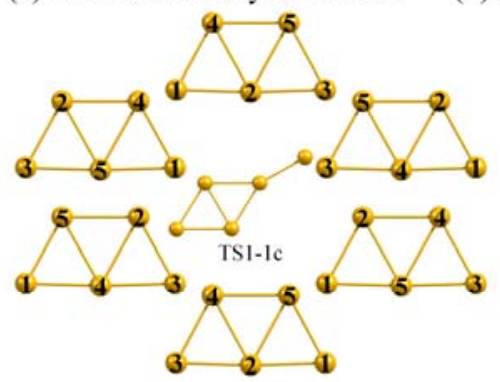

(b) The connectivity of TS1-1b

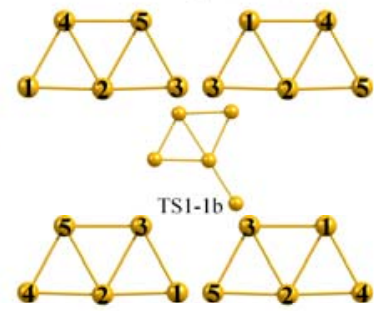

(d) The connectivity of TS1-1d

Figure 3. The NPI isomers of MIN1 connected via (a) TS1-1a, (b) TS1-1b, (c) TS1-1c, and (d) TS1-1d.

Here, we further consider the structural transformations of MIN1 through TS1-1d in Fig. 
3d. TS1-1d has a non-planar geometry where an Au atom is attached to the top of a pyramidal structure of $\mathrm{Au}_{4}$. The bottom of the $\mathrm{Au}_{4}$ pyramid is not an equilateral triangle but an isosceles triangle, and thus a point group of TS1-1d is not $C_{3 \mathrm{v}}$ but $C_{\mathrm{s}}$ symmetry. Since three different isosceles triangles are possible around an equilateral triangle, there are three NPI isomers of TS1-1d which are lying closely to each other, and there are six NPI isomers of MIN1 connected by these three TS1-1d. Figure 4 shows a connectivity relation of these three TS1-1d and six MIN1. ${ }^{18}$ As shown here, MIN1 and MIN1" are connected via TS1-1b that is located near VRT1 and VRT1" (in the same way, MIN1' and MIN1"' are connected via TS1-1b', and MIN1"'" and MIN1"'" are connected via TS1-1b"). Let's consider the $C_{3 \mathrm{v}}$ structure located at the central region of three NPI isomers of TS1-1d of $C_{\mathrm{s}}$ symmetry in a configuration space. At this $C_{3 v}$ structure, the highest occupied molecular orbital (HOMO) corresponds to a singly-occupied molecular orbital (SOMO) of $e$ representation. Since a spin multiplicity of $\mathrm{Au}_{5}$ is doublet, the electronic term of this $C_{3 \mathrm{v}}$ structure is ${ }^{2} E$, which is a doubly-degenerate state (corresponding to a conical intersection (CI) of the ground and first-excited states). It is well known that a nonlinear molecule in a degenerate electronic state loses geometrical symmetry by the first-order Jahn-Teller effect, by which the degenerated electronic states are split out (the symmetry of the molecular structure is reduced from $C_{3 \mathrm{v}}$ to $C_{\mathrm{s}}$ in the present case). The energy of the $\mathrm{CI}\left(C_{3 \mathrm{v}}\right)$ was calculated to be $3.4 \mathrm{kcal} / \mathrm{mol}$ higher than TS1-1d $\left(C_{\mathrm{s}}\right)$, indicating that the PES is flat in the region connecting TS1-1d, TS1-1d', and TS1-1d". 


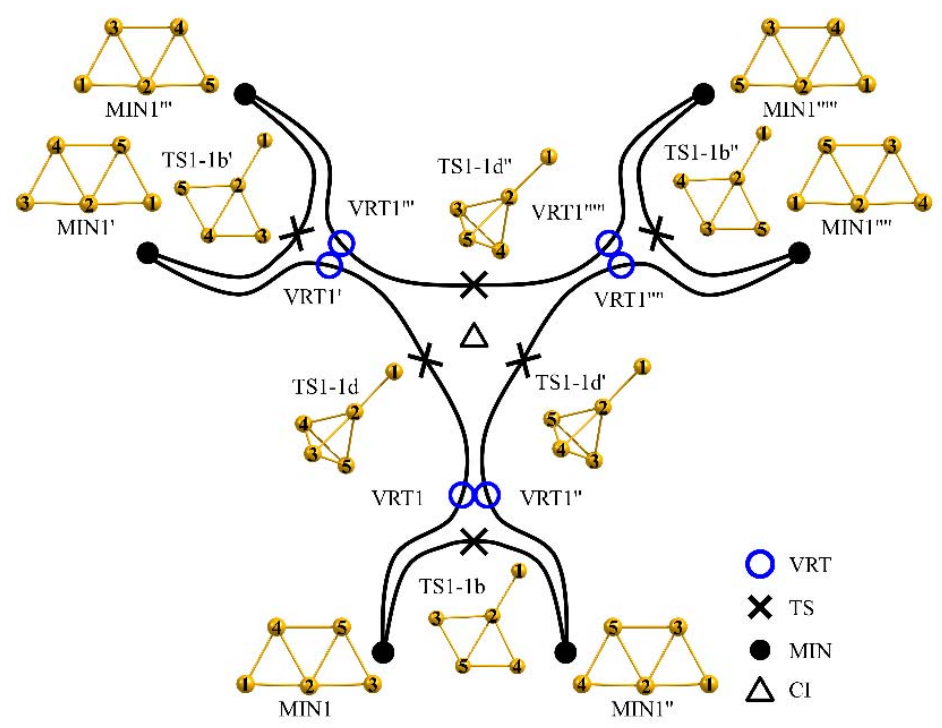

Figure 4. A schematic connectivity of TS1-1d (TS1-1d, TS1-1d', TS1-1d"; denoted by a cross mark), MIN1 (MIN1, MIN1', MIN1", MIN1"', MIN1"'", MIN1"'"; denoted by a black circle), TS1-1b (TS1-1b, TS1-1b', TS1-1b"; denoted by a cross mark), VRT1 (VRT1, VRT1', VRT1", VRT1"', VRT1"', VRT1"'"; denoted by a blue circle), and CI (denoted by a triangle). 


\section{III-B. TOF-MD simulation}

Next, we carried out the TOF analyses for $\mathrm{Au}_{5}$ based on the global reaction route map. The TOF-MD simulations were performed for the structural transformations of $\mathrm{Au}_{5}$ at the PBE/LanL2DZ level, using the SPPR program. ${ }^{35}$ The time step was set to 5 fs (determined through preliminary calculations by checking the total energy conservation), and 200 trajectories were run over 3 ps. As shown in Fig. 2, the global minimum MIN1 is connected with nine TSs where only two TSs (TS1-1d and TS1-4) have a non-planar geometry and the IRC pathways from TS1-3b, TS1-5, and TS1-1d accompany a VRT nature. Taking into account these features, TOF-MD simulations were started from TS1-1d with a kinetic energy of $5 \mathrm{kcal} / \mathrm{mol}$ where the initial velocity direction was chosen randomly in 3N-6 internal coordinates ( $N$ denotes the number of atoms). Since the energy level of TS1-1d is relatively high $(20.8 \mathrm{kcal} / \mathrm{mol})$, TOF can explore the potential energy surface in a wide region. When the molecular system has a small kinetic energy at TS, a trajectory starting from a TS has a tendency to follow the IRC pathway, reaching the minima connected by the IRC. As shown in Fig. 4, however, three TSs (TS1-1d, TS1-1d', TS1-1d") are lying very closely to each other in a configuration space, and thus, some trajectories possibly jump to the other IRCs in the initial stage, reaching not only MIN1 or MIN1', but also MIN1", MIN1"', MIN1"', or MIN1"'". Such a jump from one IRC to the other IRC could not be considered in a static analysis of the reaction route network, and can be examined only through a dynamics simulation. In recent works, Martínez-Núñez analyzed the accelerated dynamics simulations results using spectral graph theory to obtain stationary points, and observed the IRC jumps which was the basis for obtaining transition states of bimolecular reactions. ${ }^{36,37}$

The TOF was analyzed based on a distance function, $d_{i}(t)$ in Eq. (1), which measures a spatial distance from each NPI isomer of the reference structures, i.e., five minima, $14 \mathrm{TSs}$, and one VRT (VRT1 with $C_{1}$ symmetry) of Aus. In total, the number of the reference structures is 20 , and the 
number of the reference points (= the NPI isomers of the reference structures) amounts to 2130 (= $240 \times 2+120 \times 10+60 \times 7+30$ ). Using the distance functions, the branching of trajectories over the reference points, as well as the $\mathrm{Au}_{5}$ structural transformations over several different structures, can be discussed. The procedure to choose the closest reference structure $\mathbf{x}^{\mathrm{IRC}}(t)$ and to evaluate a distance function $d(t)$ is as follows:

(1) At each point on the TOF, distance functions are evaluated for all reference points.

(2) For each reference structure, the NPI isomer that gives the smallest distance is assigned.

(3) The reference structure that gives the smallest distance is chosen as the closest reference structure.

In this analysis, reference structures could be added as much as one likes. As the number of the reference structures increases, one can get a more detailed information about the dynamics from the TOF.

Hence, we start discussions of the TOF-MD results for $\mathrm{Au}_{5}$. As expected, some trajectories show one or more hopping over TS1-1d, TS1-1d', and TS1-1d" in the initial stage. According to the number of the initial hopping, the trajectories are classified to five cases: (A) no hopping (96 trajectories); (B) one hopping (74 trajectories); (C) two hopping (23 trajectories); (D) three hopping (6 trajectories); (E) four hopping (1 trajectory). Thus, almost half trajectories (96/200) go down on the PES along the IRC without hopping, while the remaining half trajectories (104/200) show hopping over TS1-1d, TS1-1d', and TS1-1d" before going down. Figure 5 shows schematic pictures for the initial routes from TS1-1d to VRT1 in the TOF in cases of (A) no hopping (96/200) and (B) one hopping (74/200), where the positions of the respective reference points are indicated in the same way as in Fig. 4. In a case of (A), most trajectories reach VRT1 or VRT1' which are located on the IRC (86/96; orange line), while a small number of trajectories reach VRT1" or VRT1"' (7/96; 
blue line). This branching indicates that the molecular system ( $\left.\mathrm{Au}_{5}\right)$ jumps from the IRC to the next IRC while descending on the PES, and such a behavior is expected from a bifurcating nature of the IRC with VRT. The branching ratio was estimated to be $7 / 86(\sim 0.08)$. The only one trajectory reaches VRT1"' (1/96; green line) which is lying in the other side of VRT1" on the next IRC. There are also two exceptional trajectories that directly reach the other stationary points, MIN3 and TS1-4, without through VRT1 (2/96; purple and red lines). In a case of (B), the trajectories initially hop from TS1-1d to TS1-1d' (37/74) or TS1-1d" (37/74), and then descend on the PES along the IRC pathway. When hopping to TS1-1d', most trajectories descend toward VRT1" (26/37) while a very small number of trajectories go toward VRT1"' (2/37). In the same way, when hopping to TS1-1d", most trajectories descend toward VRT1"' (26/37) while eight trajectories go toward VRT1"'"' (8/37). The larger rate of VRT1" and VRT1"' can be intuitively understood by considering that they are located in the nearer region from the original IRC passing through TS1-1d. There are a small number of trajectories from TS1-1d' to VRT1 and from TS1-1d" to VRT1' or VRT1.

(A) No hopping $(96 / 200)$

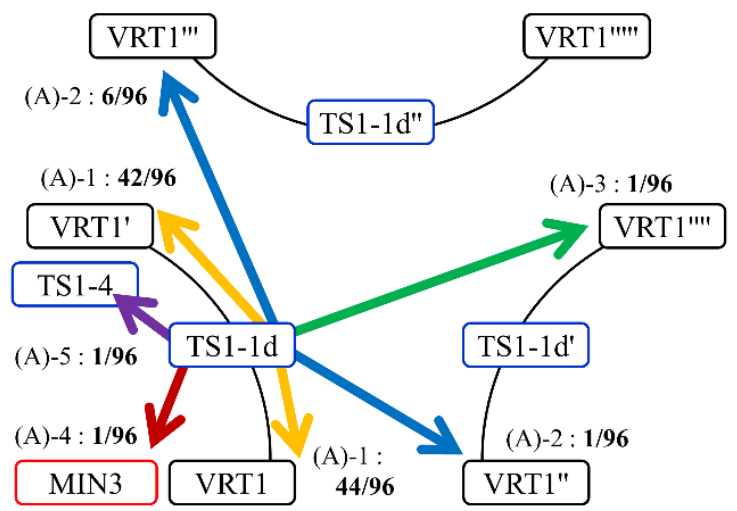

(B) One hopping $(74 / 200)$

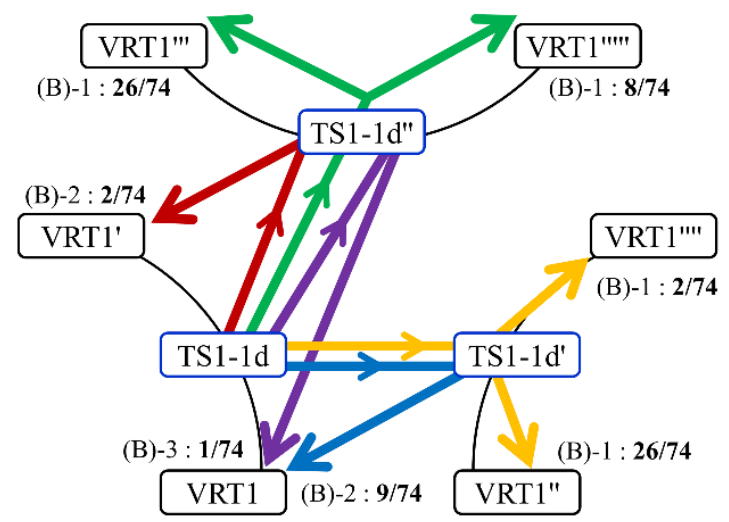

Figure 5. Schematic pictures for the initial routes from TS1-1d to VRT1 in the TOF in cases of (A) no hopping and (B) one hopping over TS1-1d, TS1-1d', and TS1-1d". 
Figure 6 shows a dendrogram of 200 trajectories from TS1-1d until reaching VRT1 where TS1-1d(n) denotes TS1-1d after the $n$th hopping over TS1-1d, TS1-1d', and TS1-1d". The number of trajectories and the averaged time to reach VRT1 for the detailed routes are also shown in parentheses. Hence, VRT1 $1_{\text {IRC }}$ denotes VRT1 directly connected with the latest TS1-1d (TS1-1d, TS1-1d', or TS1-1d"), VRT1 $1_{\text {bifur }}$ denotes VRT1 located on the IRC next to that with VRT1 1 IRC, and

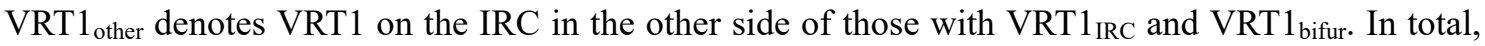
175 trajectories reach VRT1 $1_{\text {IRC }}, 21$ trajectories reach VRT $1_{\text {bifur, }}$, and two trajectories reach VRT $1_{\text {other, }}$, and thus, the branching ratio for $\mathrm{VRT} 1_{\text {bifur }} / \mathrm{VRT} 1_{\text {IRC }}$ is $21 / 175(\sim 0.12)$ which is larger than the rate for no hopping case (7/86). As the number of hopping increases, the averaged time to reach VRT $1_{\text {IRC }}$ also increases as $342 \mathrm{fs}$ (no hopping), $453 \mathrm{fs}(n=1), 583 \mathrm{fs}(n=2), 1055 \mathrm{fs}(n=3)$, and $1245 \mathrm{fs}(n=$ 4). It is interesting to note that, for no hopping and one hopping cases, the molecular system reaches VRT $1_{\text {bifur }}$ and $V R T 1_{\text {other }}$ faster than VRT1 $1_{\text {IRC }}$ on average

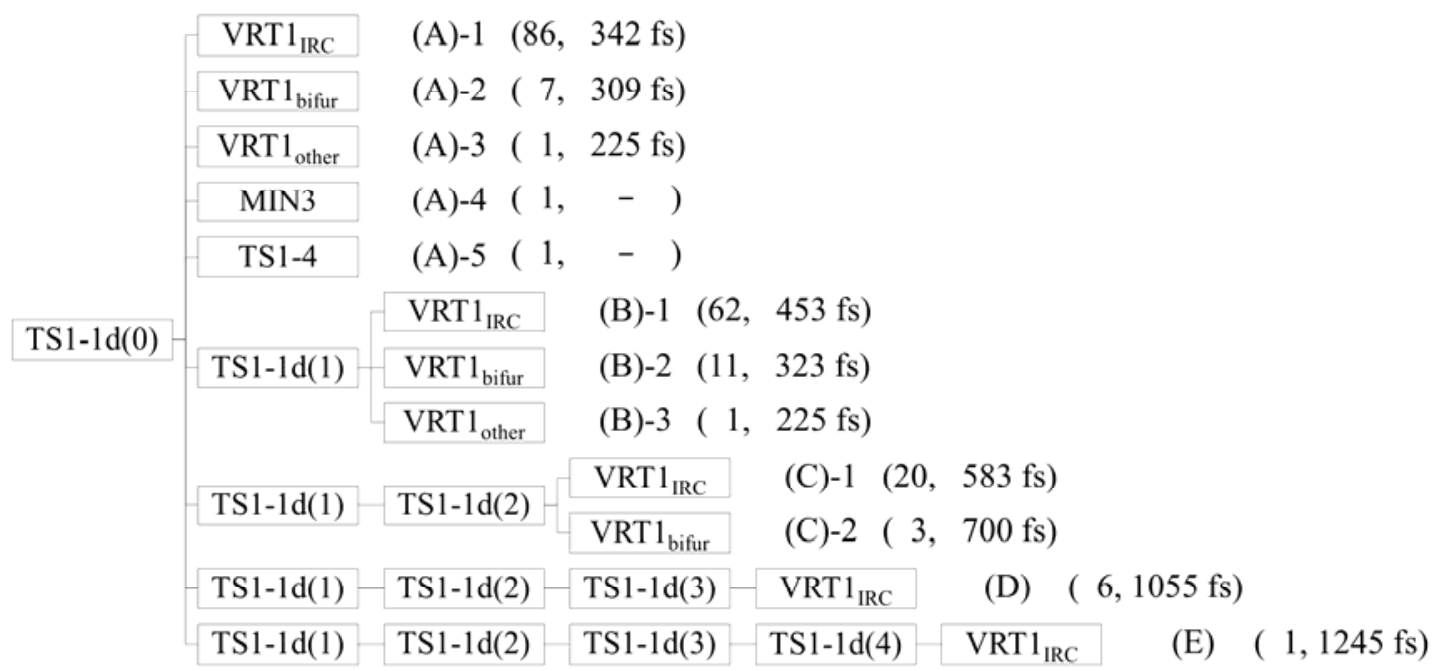

Figure 6. A dendrogram of 200 trajectories that start from TS1-1d and reach VRT1 where TS1-1d(n) denotes TS1-1d after the $n$th hopping over TS1-1d, TS1-1d', and TS1-1d". For the respective routes, 
the number of trajectories and the average time to reach VRT1 are given in parentheses.

We move to discussions of traces of TOF over 3 ps. As described above, distance functions with 2130 reference points were calculated for 601 points of each TOF (every 5 fs over 3 ps), and variations of distance functions with 20 reference structures were plotted as a function of time for 200 trajectories. Figure 7 shows six patterns of variations of distance functions along the TOF, as examples. In all cases, trajectories start from TS1-1d, and thus, a distance from TS1-1d is zero at $t=$ 0 fs. In Fig. 7(a), the trajectory descends on the PES along the IRC, and reaches VRT1 at $t \sim 500$ fs. Then, it goes through TS1-1b which is located near VRT1, and succeedingly reaches MIN1 at $t \sim$ 1200 fs. This is the most typical route, which was observed in 86 trajectories. After that, the trajectory shows continuous structural transformations among NPI isomers of MIN1 via TS1-1a with the lowest barrier height $(\sim 3.8 \mathrm{kcal} / \mathrm{mol})$. Figure $7(\mathrm{~b})$ shows the trajectory which proceeds as similar to the case (a) until reaching MIN1, but later it reaches MIN2 at $t \sim 2500$ fs via TS1-2. Among 200 trajectories, the number of trajectories reaching MIN $n$ within 3 ps is $192(n=1), 5(n=2), 1(n=3)$, $0(n=4)$, and $0(n=5)$. The small numbers in trajectories reaching MIN2 $\sim$ MIN5 are due to the insufficient energy of the molecular system $\left(\mathrm{Au}_{5}\right)$ in the present simulation. In Fig. 7(c), the trajectory shows non-planar motion throughout, and attempts to go over TS1-4 twice, but it does not reach MIN4. In Fig. 7(d), the trajectory wanders around TS1-1d with four-times hopping over TS1-1d, TS1-1d', and TS1-1d", and reaches the NPI isomer of TS1-1d other than TS1-1d, TS1-1d', and TS1-1d" (referred to as TS1-1d $\mathrm{d}_{\text {outside }}$ ) via VRT1 and TS1-1b (denoted as VRT1/TS1-1b); it does not reach MIN1 during 3 ps. Figure 7(e) and 7(f) show a very rare case where the trajectory approaches MIN3 and TS1-4, respectively, from TS1-1d directly (not via VRT1/TS1-1b), which correspond to (A)-4 and (A)-5, respectively, in Fig. 6. 

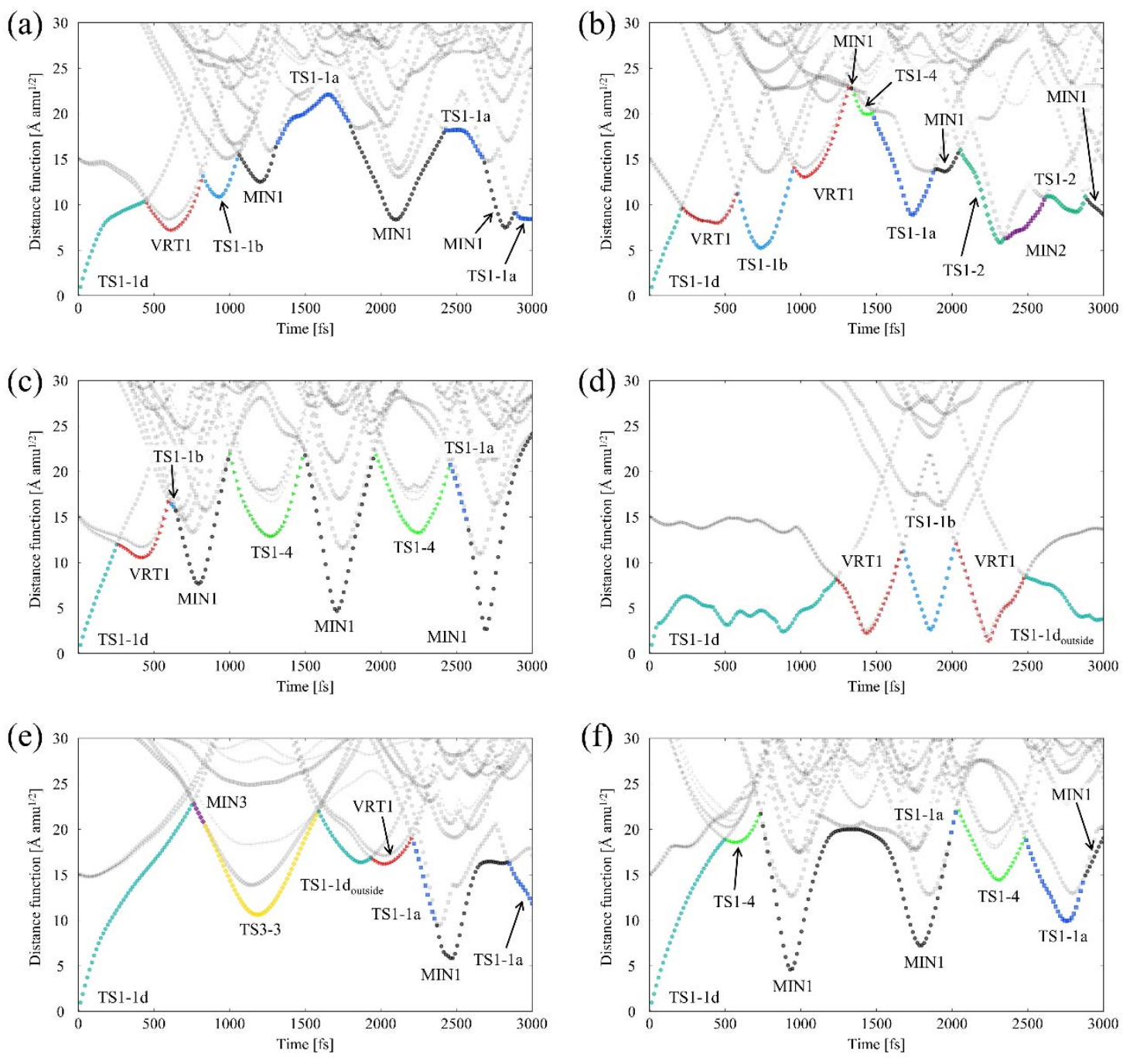

Figure 7. Variations of distance functions with reference structures as a function of time, for six trajectories.

Figure 8 shows a schematic picture of TOF routes from TS1-1d to MIN1 via VRT1/TS1-1b. As discussed above, only two trajectories do not go through VRT1/TS1-1b, so the routes of 198 trajectories are shown here. After passing VRT1/TS1-1b, trajectories approach to seven

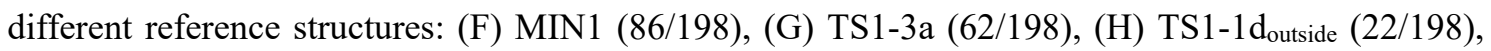


(I) TS1-1a (16/198), (J) TS1-2 (7/198), (K) TS1-4 (4/198), (L) TS1-3b (1/198). In cases of (H) (7/22) and (L) (1/1), trajectories do not reach MIN1 during 3 ps. More detailed routes from VRT1/TS1-1b to MIN1 for cases of $(\mathrm{F}) \sim(\mathrm{K})$ are described in Supporting Information.

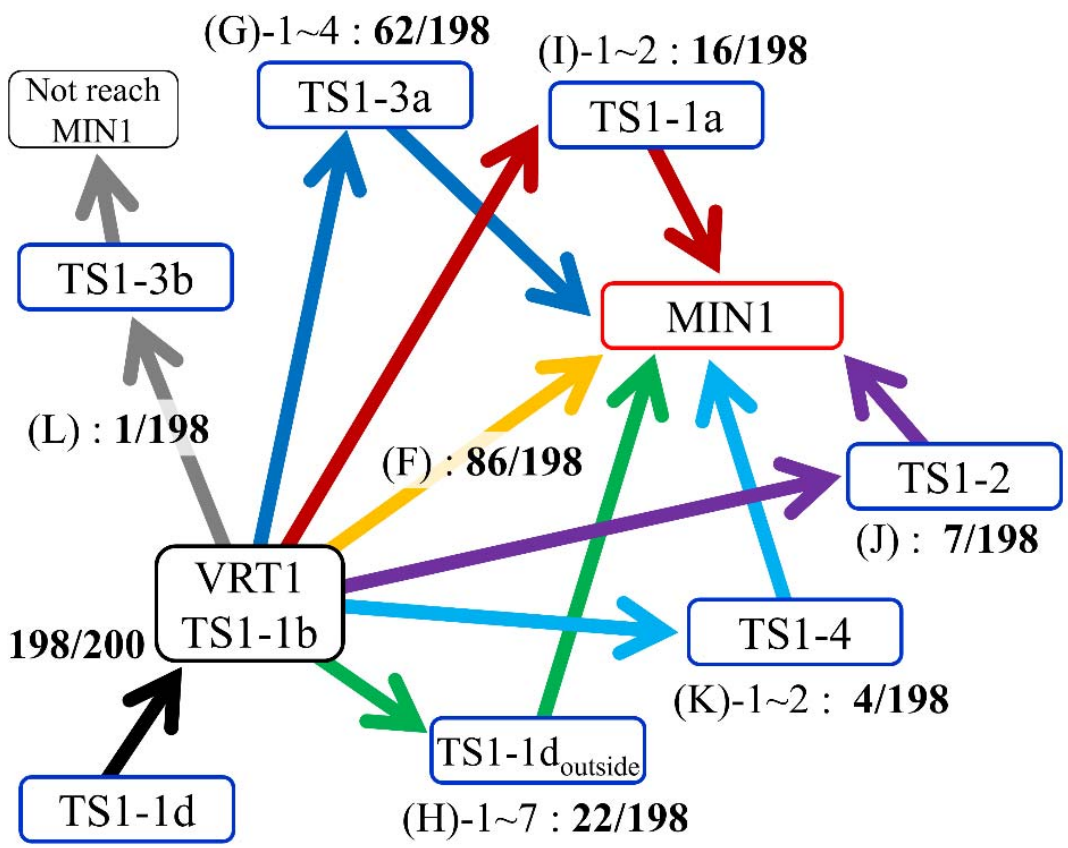

Figure 8. A schematic picture for the TOF routes from TS1-1d to MIN1 via VRT1/TS1-1b. In total 198 trajectories go through VRT1/TS1-1b. There are seven different routes, $(\mathrm{F}) \sim(\mathrm{L})$. The number of trajectories going through each route is also given.

All the TOF routes from TS1-1d to the first arrival of MIN1 are summarized in a dendrogram in Figure 9. For the respective routes, the number of trajectories and an average time to reach MIN1 are given in parentheses. As shown here, the routes of trajectories are classified into 20 patterns, and eight trajectories do not reach MIN1 during 3 ps. Most trajectories (198/200) go down along the IRC via VRT1/TS1-1b, but before reaching MIN1 the trajectories show a variety of routes going through several TSs (IRCs). Such a behavior could be classified to IRC-jump routes, caused 
by the existence of closely-lying reaction path networks in the configurational space. The existence of such a closely-lying reaction paths sometimes appear as a VRT feature in the IRC pathway. TOF-MD is a powerful tool to detect such a feature of the global reaction route map.

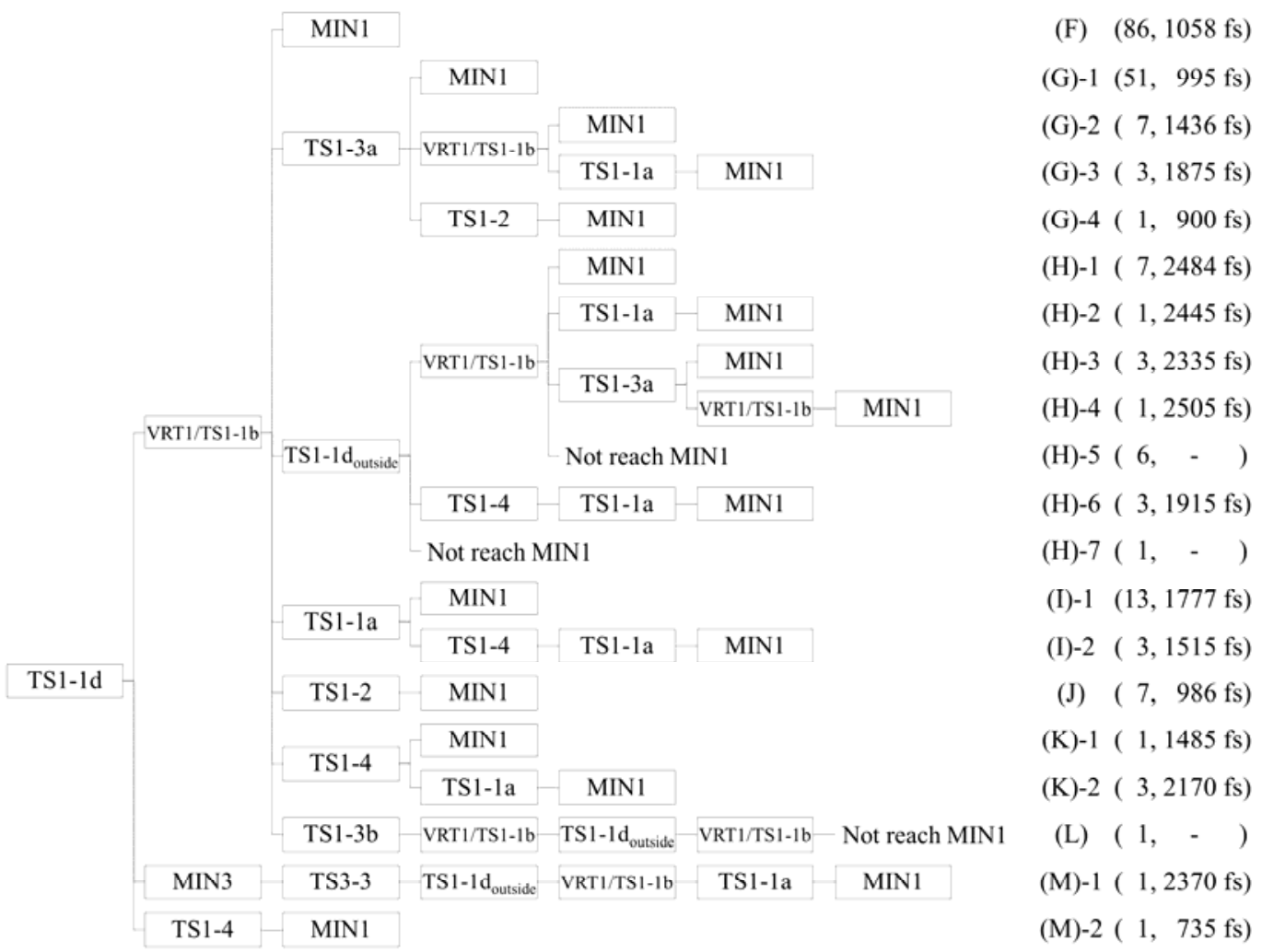

Figure 9. A dendrogram of 200 trajectories that start from TS1-1d and reach MIN1: (F) VRT1/TS1-1b $\rightarrow$ MIN1; (G) VRT1/TS1-1b $\rightarrow$ TS1-3a; (H) VRT1/TS1-1b $\rightarrow$ TS1-1d outside; $_{\text {(I) }}$ VRT1/TS1-1b $\rightarrow$ TS1-1a; (J) VRT1/TS1-1b $\rightarrow$ TS1-2; (K) VRT1/TS1-1b $\rightarrow$ TS1-4; (L) VRT1/TS1-1b $\rightarrow$ TS1-3b; (M) not through VRT1/TS1-1b. For the respective routes, the number of trajectories and an average time to reach MIN1 are given in parentheses. 


\section{CONCLUDING REMARKS}

In the present paper, we propose a new methodology to analyze TOF based on a global reaction route map. The procedure is very simple, in which a distance function was evaluated for a given molecular geometry and the reference points on the reaction pathways in $3 \mathrm{~N}$-dimensional mass-weighted coordinates, and trajectories were mapped on the IRC networks based on the distance functions. The proposed analyses were applied to structural transformations of $\mathrm{Au}_{5}$ where not only minima but also TSs and VRTs are included as the reference structures. The global reaction route map for $\mathrm{Au}_{5}$ includes $14 \mathrm{IRCs}$ and $5 \mathrm{VRT}$ points, and the respective reference structures have 240 NPI isomers in a case of $C_{1}$ symmetry. The trajectories starting from TS1-1d show a variety of branches over a lot of minima, and also 20 different reaction routes to minima are detected. A feature of IRC-jump was also observed in many trajectories.

In general, as the molecular system gets a higher energy, the movement of each atom in the molecular system could be deviated far from the IRC pathway, because the contribution from the kinetic energy increases more and more. Thus, in a high-energy dynamics, the IRC pathway will lose the role of a reference reaction pathway, but TOF itself is accurate even in such a high-energy situation. The present analysis is just to map the TOF on the global reaction route map, so the analysis provides a physical insight to dynamics even in a high-energy region, although the observation of the IRC-jump will increase. Further applications of the TOF-MD analysis are now in progress in our group. 


\section{Conflicts of interest}

There are no conflicts of interest to declare.

\section{ACKNOWLEDGMENT}

This work was supported by JSPS KAKENHI with Grant Number 16KT0047, and partly supported by MEXT as "Priority Issue on Post-K computer" (Development of new fundamental technologies for high-efficiency energy creation, conversion/storage and use). YH is supported by JST for PRESTO (Grant Number JPMJPR16N8). A part of calculations was performed using the Research Center for Computational Science, Okazaki, Japan. 


\section{REFERENCES}

(1) Gordon, M. S.; Chaban, G.; Taketsugu, T. Interfacing electronic structure theory with dynamics. $J$. Phys. Chem. 1996, 100, 11512-11525.

(2) Sun, L.; Song, K.; Hase, W. L. A $\mathrm{S}_{\mathrm{N}} 2$ reaction that avoids its deep potential energy minimum. Science 2002, 296, 875-878.

(3) Pratihar, S.; Ma, X.; Homayoon, Z.; Barnes, G. L.; Hase. W. L. Direct Chemical Dynamics Simulations. J. Am. Chem. Soc. 2017, 139, 3570-3590.

(4) R. Car, R.; Parrinello, M. Unified Approach for Molecular Dynamics and Density-Functional Theory. Phys. Rev. Lett. 1985, 55, 2471-2474.

(5) Mann, D. J.; Hase, W. L. Ab Initio Direct Dynamics Study of Cyclopropyl Radical Ring-Opening. J. Am. Chem. Soc. 2002, 124, 3208-3209.

(6) Ben-Nun, M.; Martínez, T. Ab initio quantum molecular dynamics. Adv. Chem. Phys., 2002, 121 439-512.

(7) Taketsugu, T.; Tajima, A.; Ishii, K.; Hirano, T. Ab initio direct trajectory simulation with nonadiabatic transitions of the dissociative recombination reaction $\mathrm{HCNH}^{+}+\mathrm{e}^{-} \rightarrow \mathrm{HNC} / \mathrm{HCN}+\mathrm{H}$. Astrophys. J. 2004, 608, 323-329.

(8) Ootani, Y.; Satoh, K.; Nakayama, A.; Noro, T.; Taketsugu, T. Ab initio molecular dynamics simulation of photoisomerization in azobenzene in the $\mathrm{n} \pi^{*}$ state. J. Chem. Phys. 2009, 131, 194306.

(9) Fukui, K. A formulation of the reaction coordinate. J. Phys. Chem. 1970, 74, 4161-4163.

(10) Maeda, S.; Harabuchi, Y.; Ono, Y.; Taketsugu, T.; Morokuma, K. Intrinsic reaction coordinate: calculation, bifurcation, and automated search, Int. J. Quantum Chem. 2015, 115, 258-269.

(11) Kato, S.; Morokuma, K. Potential-energy characteristics and energy partitioning in chemical-reactions - ab initio MO study of 4-centered elimination-reaction $\mathrm{CH}_{3} \mathrm{CH}_{2} \mathrm{~F} \rightarrow$ $\mathrm{CH}_{2}=\mathrm{CH}_{2}+$ HF. J. Chem. Phys. 1980, 73, 3900-3914. 
(12) Taketsugu, T.; Gordon, M. S. Dynamic reaction-path analysis based on an intrinsic reaction coordinate. J. Chem. Phys. 1995, 103, 10042-10049.

(13) Taketsugu, T.; Gordon, M. S. Reaction path hamiltonian based on a reaction coordinate and a curvature coordinate. J. Chem. Phys. 1996, 104, 2834-2840.

(14) Ohno, K.; Maeda, S. A scaled hypersphere search method for the topography of reaction pathways on the potential energy surface. Chem. Phys. Lett. 2004, 384, 277-282.

(15) Maeda, S.; Taketsugu, T.; Morokuma, K.; Ohno, K. Anharmonic downward distortion following for automated exploration of quantum chemical potential energy surfaces. Bull. Chem. Soc. Jpn. 2014, $87,1315-1334$.

(16) Maeda, S.; Ohno, K.; Morokuma, K. Systematic exploration of the mechanism of chemical reactions: the global reaction route mapping (GRRM) strategy using the ADDF and AFIR methods. Phys. Chem. Chem. Phys. 2013, 15, 3683-3701.

(17) Maeda, S.; Harabuchi, Y.; Takagi, M.; Taketsugu, T.; Morokuma, K. Artificial force induced reaction (AFIR) method for exploring quantum chemical potential energy surfaces. Chem. Rec. 2016, 16, $2232-2248$.

(18) Harabuchi, Y.; Ono, Y.; Maeda, S.; Taketsugu, T. Analyses of bifurcation of reaction pathways on a global reaction route map: a case study of gold cluster Aus. J. Chem. Phys. 2015, 143, 014301.

(19) Quapp, W. Comment on 'Analyses of bifurcation of reaction pathways on a global reaction route map: a case study of gold cluster $\mathrm{Au}_{5}^{\prime}$. J. Chem. Phys. 2015, 143, 177101.

(20) Harabuchi, Y.; Ono, Y.; Maeda, S.; Taketsugu, T. Response to "Comment on 'Analyses of bifurcation of reaction pathways on a global reaction route map: a case study of gold cluster $\mathrm{Au}_{5}{ }^{\prime}$ " [J. Chem. Phys. 2015, 143, 177101]. J. Chem. Phys. 2015, 143, 177102.

(21) Kabsch, W. A solution for the best rotation to relate two sets of vectors. Acta Cryst. 1976, A32, 922-923. 
(22) Longuet-Higgins, H. C. The symmetry groups of non-rigid molecules. Mol. Phys. 1963, 6, 445-460.

(23) Taketsugu, T.; Wales, D. J. Theoretical study of rearrangements in water dimer and trimer. Mol. Phys. 2002, 100, 2793-2806.

(24) Frisch, M. J.; Trucks, G. W.; Schlegel, H. B.; Scuseria, G. E.; Robb, M. A.; Cheeseman, J. R.; Scalmani, G.; Barone, V.; Mennucci, B.; Petersson, G. A.; Nakatsuji, H.; Caricato, M.; Li, X.; Hratchian, H. P.; Izmaylov, A. F.; Bloino, J.; Zheng, G.; Sonnenberg, J. L.; Hada, M.; Ehara, M.; Toyota, K.; Fukuda, R.; Hasegawa, J.; Ishida, M.; Nakajima, T.; Honda, Y.; Kitao, O.; Nakai, H.; Vreven, T.; Montgomery, J. A.; Jr.; Peralta, J. E.; Ogliaro, F.; Bearpark, M.; Heyd, J. J.; Brothers, E.; Kudin, K. N.; Staroverov, V. N.; Kobayashi, R.; Normand, J.; Raghavachari, K.; Rendell, A.; Burant, J. C.; Iyengar, S. S.; Tomasi, J.; Cossi, M.; Rega, N.; Millam, J. M.; Klene, M.; Knox, J. E.; Cross, J. B.; Bakken, V.; Adamo, C.; Jaramillo, J.; Gomperts, R.; Stratmann, R. E.; Yazyev, O.; Austin, A. J.; Cammi, R.; Pomelli, C.; Ochterski, J. W.; Martin, R. L.; Morokuma, K.; Zakrzewski, V. G.; Voth, G. A.; Salvador, P.; Dannenberg, J. J.; Dapprich, S.; Daniels, A. D.; Farkas, O.; Foresman, J. B.; Ortiz, J. V.; Cioslowski, J.; Fox, D. J., Gaussian 09, revision a.1, Gaussian, Inc., Wallingford CT, 2009.

(25) S. Maeda; Y. Harabuchi; Y. Sumiya; M. Takagi; M. Hatanaka; Y. Osada; T. Taketsugu; K. Morokuma; Ohno, K., GRRM (a developmental version), Hokkaido University, 2017. (see http://grrm.chem.tohoku.ac.jp/GRRM/index_e.html [accessed on March 3, 2016], GRRM14)

(26) Pyykko, P. Theoretical chemistry of gold. Angew Chem. Int. Ed. Engl. 2004, 43, 4412-56.

(27) Valtazanos, P.; Ruedenberg, K. Bifurcations and transition-states. Theor. Chim. Acta. 1986, 69, 281-307.

(28) Baker, J.; Gill, P. M. W. An algorithm for the location of branching points on reaction paths. J. Comput. Chem. 1988, 9, 465-475.

(29) Taketsugu, T.; Tajima, N.; Hirao, K. Approaches to bifurcating reaction path. J. Chem. Phys. 1996, 
$105,1933-1939$.

(30) Yanai, T.; Taketsugu, T.; Hirao, K. Theoretical study of bifurcating reaction paths. J. Chem. Phys. 1997, 107, 1137-1146.

(31) Shaik, S.; Danovich, D.; Sastry, G. N.; Ayala, P. Y.; Schlegel, H. B. Dissociative electron transfer, substitution, and borderline mechanisms in reactions of ketyl radical anions. Differences and difficulties in their reaction paths. J. Am. Chem. Soc. 1997, 119, 9237-9245.

(32) Harabuchi, Y.; Taketsugu, T. A significant role of the totally symmetric valley-ridge inflection point in the bifurcating reaction pathway. Theor. Chem. Acc. 2011, 130, 305-315.

(33) Harabuchi, Y.; Nakayama, A.; Taketsugu, T. Trifurcation of the reaction pathway. Comp. Theor. Chem. 2012, 1000, 70-74.

(34) Bofill, J. M.; Quapp, W. Analysis of the valley-ridge inflection points through the partitioning technique of the hessian eigenvalue equation. J. Math. Chem. 2013, 51, 1099-1115.

(35) Harabuchi, Y.; Okai, M.; Yamamoto, R.; Tsutsumi, T.; Ono, Y.; Taketsugu, T., SPPR, a developmental version at hokkaido university, Sapporo, Japan, 2017.

(36) Martínez-Núñez, E. An automated method to find transition states using chemical dynamics simulations. J. Comput. Chem. 2015, 36, 222-234.

(37) Martínez-Núñez, E. An automated transition state search using classical trajectories initialized at multiple minima. Phys. Chem. Chem. Phys. 2015, 17, 14912-14921. 